\title{
COOPERATION, COMPETITION, AND TEAM PERFORMANCE: TOWARD A CONTINGENCY APPROACH
}

\author{
BIANCA BEERSMA \\ University of Amsterdam \\ JOHN R. HOLLENBECK \\ STEPHEN E. HUMPHREY \\ Michigan State University \\ HENRY MOON \\ Emory University \\ DONALD E. CONLON \\ DANIEL R. ILGEN \\ Michigan State University
}

\begin{abstract}
This study examined whether the relationship between reward structure and team performance is contingent upon task dimension, team composition, and individual performance level. Seventy-five four-person teams engaged in a simulated interactive task in which reward structure was manipulated. A competitive structure enhanced one task dimension, speed, whereas a cooperative structure enhanced accuracy. Teams with extroverted and agreeable members performed better under the cooperative structure, whereas teams low on these orientations performed better under the competitive structure. Finally, reward structure had more impact on team members with low performance.
\end{abstract}

The degree to which organizations should emphasize cooperation or competition among the members of work teams is an age-old controversy. Competitive systems embody equity norms and emphasize performance differences among team members, typically rewarding individuals with high performance and/or imposing sanctions on those with low performance. Therefore, some believe that competition promotes efficiency and innovation because it stimulates individuals to outperform each other by working faster, or "smarter," or cheaper, and the belief is that this activity will serve the long-term needs of their organization. Others believe that intrateam competition is de-

This research was conducted while the first author studied at Michigan State University on a Fulbright Graduate Student Scholarship, and we thank the Netherlands America Commission for Educational Exchange gratefully for their support. Henry Moon was also at Michigan State University when the research was conducted.

Grant N00014-99-1-0983 from the Cognitive and Neural Sciences Division of the Office of Naval Research financially supported this research in part. Although support for this work is gratefully acknowledged, the ideas expressed herein are those of the authors and are not necessarily endorsed by the funding agencies. structive. In competing, individuals or subgroups place their own goals above those of the larger organization, and the gains achieved by one are often obtained at the expense of another. For this reason, some argue that the needs of the larger organization are better met by employing cooperative reward structures. Cooperative systems embody equality norms and emphasize group accomplishments. They emphasize minimizing distinctions among group members (that is, distinctions based on performance) because these distinctions may impede teamwork, information sharing, and helping.

The inherent tension between competitive and cooperative reward structures has become particularly salient in contemporary organizations because many of these organizations are trying to make the transition from individual-based structures to team-based structures (Allred, Snow, \& Miles, 1996). Some organizations that have transitioned into team-based structures over the last several years have left their reward structures unchanged. This lack of change resulted in a failure of teambased work to result in the supposed benefits (Hackman, 1998). Indeed, the consensus in the scientific literature regarding competitive and collaborative rewards structures is quite clear in its sup- 
port for competitive allocations when people are working independently, but collaborative allocations when people are interdependent (Deutsch, 1949; Miller \& Hamblin, 1963; Rosenbaum et al., 1980; Stanne, Johnson, \& Johnson, 1999; Wageman, 1995). The general logic underlying this prescription is that collaborative reward allocations promote trust, cohesiveness, and mutually supportive behavior among team members, which in turn promote performance, and this general theme is reiterated in almost all organizational behavior textbooks (cf. Ivancevich \& Matteson, 1999).

However, the simple notion that rewards for members of interdependent teams should be collaboratively based ignores the fact that even within interdependent groups, any single task can be broken down into subtasks that are evaluated by different standards. Over 100 years ago, Woodworth documented convincingly that on a task of any complexity, speed and accuracy are two separateand, in some cases, negatively related-aspects of a single task (Woodworth, 1899). Subsequent research on the speed-accuracy distinction has made it clear that the two task dimensions have very different antecedents (Elliott, Helsen, \& Chua, 2001), and hence the simple notion that collaborative rewards promote both the speed and accuracy of teams seems unlikely to be correct.

Moreover, people have different traits and dispositions, and even within an interdependent team context, individuals make decisions for themselves. The traits and dispositions of these individuals will affect their own behavior in a way that may override or run counter to the reward system under which they work. For example, team-based rewards are designed to promote trust and collaboration, but for over 70 years, psychologists have noted consistent individual differences in the degree to which people are naturally trusting and collaborative (McDougall, 1932). Indeed, in the best-established framework for understanding traits, the five-factor model, two of the five factors (agreeableness and extroversion) are devoted to people's interpersonal orientation (McCrae \& Costa, 1997). As with the speed-accuracy distinction, the idea that one type of reward system-a collaborative one-promotes team performance regardless of the interpersonal orientation of team members again seems unlikely to be true.

Finally, although it is true that organizations that change to team-based structures without changing their reward systems encounter problems, it also has to be recognized that even where organizations change their reward structures to be more in line with team-based work, new problems often arise (Ezzamel \& Willmot, 1998). In particular, in most groups, the performance levels of individual members vary to some degree, and collaborative rewards discount these differences. Psychologists have recognized the "social loafing" phenomenon among poor performers in groups for many years (Latane, Williams, \& Harkin, 1979), and this problem, perhaps more than any other, underlies the reluctance about and resistance to team-based structures that many individuals express. Ironically, one of the well-known prescriptions for avoiding social loafing is to identify individual contributions to a group's performance and reward or punish these contributions accordingly (Miles \& Greenberg, 1993). If this prescription is valid, it calls into question the generic idea that in interdependent teams, collaborative reward structures are going to be most effective for all individuals.

With these considerations in mind, we sought in this study to test the generalizability of the traditionally accepted recommendation to use collaborative reward structures in interdependent teams. Specifically, our goal was to develop a contingency model of reward structures that encompasses the idea that even in interdependent team contexts, the relationship between the reward allocation structure and team performance will be contingent upon several variables related to the task, the composition, and the individual members of a team. More specifically, the contingency model we developed focuses on speed versus accuracy as a crucial distinction when one considers the nature of a task, interpersonal orientation as being an important factor when one considers a team's composition, and relative performance level as the central factor influencing a reward structure's impact on different team members.

Given the current state of consensus, questioning the inherent value of collaborative reward allocations in interdependent team contexts may seem heretical. However, even Stanne and colleagues (1999), who meta-analytically documented the advantages of collaborative rewards, recognized the need for more refined theorizing regarding the two types of reward structures, noting that "more effort needs to be focused on conceptualizing the essential elements of competition and clarifying the conditions under which competition may be effectively used" (Stanne et al., 1999: 148). The present study is clearly in line with this recommendation.

\section{THEORY AND HYPOTHESES}

\section{Defining Cooperative and Competitive Situations}

In his goal interdependence theory of cooperation and competition, Deutsch (1949) argued that 
people's beliefs about how their goals are related determine the way in which they interact, which in turn affects their performance and group cohesiveness. Central to this theory is the categorization of situations that create cooperative or competitive orientations within the people involved.

When a situation is structured cooperatively, there are positive correlations among team members' rewards, but when a situation is structured competitively, there are negative correlations among team members' rewards. According to goal interdependence theory, cooperatively structured situations create perceptions of shared fate and promote supportive behavior, whereby each group member looks out for the interests of the others. In addition, insights and lessons learned by one member are shared so that all can benefit vicariously from others' experiences. On the other hand, rather than share information and experience, people placed in competitive structures tend to keep valuable information proprietary. Moreover, rather than supporting each other, people placed in competitive reward structures may be motivated to impair the progress of others in an effort to gain positive advantage.

Since the formulation of Deutsch's theory, many studies have investigated the effects of cooperative and competitive rewards. Most of these studies have focused on differences between tasks and sought to determine how to match reward structures with various types of tasks. Meta-analyses of these studies have indicated that cooperative structures are far superior for eliciting group performance when the means interdependence of a task is high. "Means interdependence" is the degree to which the task that one member of a team faces is affected by the performance of others on the team (and hence requires coordination). However, competitive structures have been found to be slightly superior when people work on "means-independent" tasks, the completion of which requires little if any coordination between team members (Stanne et al., 1999). Thus, the theory and the empirical data associated with goal interdependence theory suggest that the reward structure employed in a given context needs to match the task at hand. Specifically, high-interdependence tasks should be paired with cooperative rewards, and low-interdependence tasks should be paired with competitive rewards.

\section{Dimensions of the Task: The Differing Impacts of Reward Structures on Speed and Accuracy}

Although documenting interaction effects between tasks is important, there are also within-task issues that need to be considered when reward structures are designed. Most complex tasks are multidimensional and correspondingly place multiple demands on role incumbents. At the very least, even after one has classified a task as requiring a high or low level of interdependence (a between-tasks consideration), the task itself can still be differentiated in terms of whether it demands speed, or accuracy, or both speed and accuracy in its execution.

Most complex tasks require some degree of both speed and accuracy, but there are trade-offs that make meeting both of these task requirements at the same time difficult (Woodworth, 1899; Elliott et al., 2001). That is, a manufacturing team can work quickly to produce a large number of products, but these products may have more defects than what might have been the case if the team worked slowly and carefully and produced only a small number of products. This type of speed-accuracy trade-off is ubiquitous in complex tasks. For example, a pit crew in an automobile race needs to quickly repair and maintain the car, but at the same time it has to make sure that all the necessary repairs are performed in order to avoid future mishaps. Air traffic controllers need to work quickly with pilots and ground controllers to keep arrivals and departures on time, but at the same time, they also have to ensure that safety standards are being met. Emergency medical teams need to work quickly to stabilize patients, but at the same time, they should not make any errors that may endanger the future health of the patients. Weapons directors in military strike teams need to work quickly to exploit windows of opportunity in the enemy's defenses, while at the same time minimizing "friendly fire" casualties.

Because the trade-off between the speed of task execution and the accuracy of task execution is apparent in so many team tasks, it is important to note that research concerning the performance of individuals working alone on independent tasks has shown that different reward structures influence speed and accuracy differently. That is, in a meta-analysis of 39 studies examining the impact of financial incentives on individual performance, Jenkins, Mitra, Gupta, and Shaw (1998) showed that financial incentives had a much stronger impact on tasks in which performance was measured in terms of speed rather than accuracy. Jenkins and colleagues speculated that incentives influence speed more than accuracy because speed is more sensitive to effort and hence under the control of an individual to a greater extent. Accuracy, on the other hand, may require skills or abilities that the individual simply may not possess. 
Jenkins and colleagues' (1998) results make it clear that even after the level of interdependence a particular task requires is diagnosed (in the case of their study, interdependence was low, with individuals working alone), the job of determining the appropriate reward structure is still unfinished. One still needs to consider the dimension of the task that is most crucial (speed or accuracy). Analogously, even after determining that a task is high in interdependence, and hence needs to be performed by a team, one may still need to consider whether one is going to emphasize speed or accuracy of task execution. Unfortunately, the question of how various rewards influence the speed and accuracy of interdependent teams could not be established in the Jenkins et al. study because there were no studies of teams that examined within-task differences in the quantity versus the quality of production.

There is some evidence that different reward structures differently influence different aspects of a team's task, although the research providing this evidence did not focus on contrasting speed and accuracy. Beersma and De Dreu (2003) showed that teams that worked under a cooperative structure tended to perform better on the "convergent" aspects of a creative team task (for instance, generating feasible ideas), while teams that worked under a competitive structure tended to perform better on the "divergent" aspects of the task (such as generating original ideas). Thus, when a team's task is conceptualized as multifaceted, much more can be learned about when and why different reward structures should be used or avoided.

The same types of within-task differences seen in the studies of individuals that were meta-analyzed by Jenkins and coauthors (1998) and in the teams studied by Beersma and De Dreu (2003) might be manifested with respect to reward structures in team contexts. For example, according to goal interdependence theory, even though they are working as part of an interdependent team, people within teams who are exposed to a competitive reward structure may feel less cohesive. As such, they may react like the individuals studied by Jenkins and colleagues and increase the speed with which they work. Moreover, a great deal of research documents that people working in groups generally take longer to complete tasks than would individuals working alone (Levine \& Moreland, 1998), and this time lag would only be exacerbated by reward structures that promote discussion, collaboration, and information sharing. If this were true, then in interdependent teams, the speed of performance might be higher when rewards are structured competitively rather than cooperatively.
Alternatively, given that financial incentives have not been shown to have an effect on the accuracy of performance when individuals work alone (Jenkins et al., 1998), it seems unlikely that competitive rewards would promote this aspect of task performance in teams. However, the collaborative nature of a group's interaction when its members work under a cooperative reward structure would seem to have the potential to enhance accuracy. In many complex team tasks, accuracy is a function of task-relevant knowledge. Under a cooperative reward structure, the team member with the most knowledge can share what he or she knows with the team members with the least knowledge, who can then use this knowledge to assess their own work processes in a way that allows them to perform higher-quality work than they could have managed on their own. If this were true, then the accuracy of performance might be higher when rewards are structured cooperatively than when they are structured competitively. Taken together, these arguments lead to our first hypothesis:

Hypothesis 1. The relationship between reward structure and performance is contingent upon the dimension of a task: speed is enhanced by competitive reward structures, whereas accuracy is enhanced by cooperative reward structures.

\section{Team Composition: The Impact of Reward Structures Composed of Interpersonally Skilled and Interpersonally Unskilled Members}

Just as one may need to address concerns regarding speed versus accuracy before choosing a reward structure, one may also need to consider the dispositional characteristics of team members prior to choosing a reward structure. That is, there may need to be a fit between the traits of members and the reward structure used with a team. The argument that there needs to be a fit between people and their work environment is consistent with a long line of theorizing on diverse aspects of organizational behavior in general (Kristof, 1996) and on teams in particular (Hollenbeck et al., 2002). Many of the more recent investigations into fit have exploited the emergence of the five-factor model as a robust, well-grounded, and culturally generalizable conceptual framework and measurement system for conducting research on individual differences (McCrae \& Costa, 1997). However, to date, very little research has addressed the question of what types of people function best under cooperative and competitive reward structures (Wageman, 1995), and no research whatsoever has approached this issue employing the five-factor model. 
Because goal interdependence theory focuses on how a reward structure influences the interaction patterns between team members, traits within the five-factor model that relate to interpersonal orientation are especially relevant in this context. That is, the five traits that are identified by this approach can be conceived as composing a "circumplex" (a circular configuration) in which certain pairs of traits are more theoretically proximal to each other than are others. Costa and McCrae (1992) defined the interpersonal plane of the circumplex as being comprised of extroversion and agreeableness. Because these traits influence interaction patterns between people, they seem ideally suited to being integrated with goal interdependence theory in an effort to understand how people are likely to react to reward structures.

More specifically, Costa and McCrae (1992) described extroverts as "liking people and working in groups." In contrast, introverts are "reserved and independent" and "tend to dislike and avoid social stimulation" (Costa \& McCrae, 1992: 15). It is easy to see how this dispositional characteristic could support or contradict an existing reward structure. Cooperative reward structures reinforce the proclivities of extroverts but work against the proclivities of introverts. In contrast, competitive structures fit the interpersonal style of introverts better than they fit the style of extroverts.

Agreeableness is the second trait that Costa and McCrae placed in the "interpersonal plane" of the five-factor model. Those high in agreeableness are described as "fundamentally altruistic, sympathetic to others, eager to help and be helped in return. By contrast, the disagreeable person is egocentric, skeptical of others' intentions, and competitive rather than cooperative (Costa \& McCrae, 1992: 15). Clearly, as in the case of extroversion, cooperative reward structures reinforce the dispositional tendencies of highly agreeable people but work against those of people who are low in agreeableness, and vice versa for competitive reward structures.

Thus, both of these traits associated with the interpersonal plane of the five-factor model should be relevant for creating a fit between a reward structure and a team's members. More specifically, we propose

Hypothesis 2a. The relationship between reward structure and performance is contingent upon the extroversion of team members: the performance of teams whose members are high on extroversion is higher when reward structures are cooperative, whereas the performance of teams whose members are low in extroversion is higher when reward structures are competitive.

Hypothesis $2 b$. The relationship between reward structure and performance is contingent upon the agreeableness of team members: the performance of teams whose members are high on agreeableness is higher when reward structures are cooperative, whereas the performance of teams whose members are low in agreeableness is higher when reward structures are competitive.

\section{The Relative Standing of Team Members: The Impact of Reward Structures on the Best and Worst Performers}

In any team, it is unlikely that all members are exactly equal in their ability or willingness to contribute to the team's overall performance level. Recent theoretical work has explicitly addressed how the member with the lowest performance influences a team (LePine \& Van Dyne, 2001). Cooperative and competitive reward structures are both posited to influence this issue. The first, positive proposition is that the member with the most knowledge will share that knowledge with the least knowledgeable person and thereby raise the latter's performance higher than it would have been had that individual been working alone. This phenomenon is a "training response," in the words of LePine and Van Dyne (2001). The second, negative proposition is that, under a cooperative structure, members, who have the opportunity to free ride on the accomplishments of the other team members, will engage in social loafing (Latane et al., 1979). Social loafing can create animosity among team members and restrict group output.

Interestingly, the traditional "cure" for social loafing is to isolate individual contributions to a group's performance and reward or punish people on the basis of these contributions (LePine and Van Dyne [2001] called this a "motivate response"). Of course, rewarding and punishing individual contributions implies having used a competitive reward structure aimed at increasing the performance of the potentially poorest performers. The ironic part of this analysis, of course, is that each of the two mutually exclusive reward structures is seen as the solution to enhancing the performance of group members viewed as likely to be the worst performers.

One way to perhaps resolve this discrepancy is to revisit the distinction between speed and accuracy. When contributors to the social loafing literature have focused on individuals with the lowest performance in a group, the presumption has been that 
they are failing to put forth effort. When authors advocating interdependence have focused on individuals with the lowest performance, the presumption seems to be that they lack knowledge or information. Both approaches could be valid if competitive reward structures work to increase the speed (effort) of team members, but cooperative reward structures work to increase their accuracy (knowledge). This formulation would be consistent with Jenkins, Mitra, Gupta, and Shaw's (1998) argument that an individual may have more control over the speed of task performance, which is largely a function of effort, than over the accuracy of task performance, which is more closely related to skill.

Moreover, the impact of the two different reward structures on the poorest performer in a group (when performance is individually assessed) is likely to be stronger than the structures' impact on the group's best performer for several reasons. First, the group decision making literature makes it very clear that the accuracy of groups' decisions tends to be much higher than that of their average members but is rarely better than that of their best members (Levine \& Moreland, 1998). Thus, from a goal interdependence perspective, the member of a team who has the lowest amount of knowledge has a great deal more to gain from collaboration the member with the most knowledge. Similarly, from a social loafing perspective, the team's slowest member has much more to fear from having this incapacity made public than the team's fastest member has to fear from having this ability made public. These arguments suggest that reward structure will be observed to have more strongly influenced the performance of the members of a team who have the lowest individual performance. Therefore, we propose

Hypothesis 3. The impact of a team's reward structure on the speed and accuracy of the team's poorest performer is stronger (in terms of explained variance) than the impact of the reward structure on the speed and accuracy of the best performer.

\section{METHODS}

\section{Research Participants}

Three hundred business students at Michigan State University were arrayed into 75 four-person work teams. Sixty percent of our sample members were male, and approximately 90 percent were Caucasian. In return for their participation, participants earned class credit and were eligible for cash prizes ( $\$ 10$ per student) based upon their perfor- mance (see "Reward structure" under "Manipulations and Measures," below).

\section{Task and Objectives}

Participants engaged in a dynamic and networked computer simulation. The task was a modified version of a simulation developed for the U.S. Department of Defense for research and training, Michigan State University Distributed Dynamic Decision Making (MSU-DDD). The version of the task used here was developed for teams of two to five members with little or no military experience.

The geographical space and mission. Figure 1 depicts the grid used in MSU-DDD. This grid was partitioned in several ways. First, four geographic quadrants of equal area (NW, NE, SW, SE) were defined, and each area was assigned to one team member, who was called a "decision maker" (hence the abbreviation "DM" in Figure 1). The grid was also divided into three zones that varied on the extent of protection from penetration by unfriendly forces they needed. The regions were labeled "neutral," "restricted" (a 12-by-12 grid in the center), and "highly restricted" (a 4-by-4 grid in the center of the restricted zone). The team's mission was to monitor this air and ground space, keeping unfriendly forces from moving into the restricted areas, while at the same time allowing friendly forces to move about freely. Radar representations of these forces moving through the geographic space monitored by the team were known as "tracks."

Each decision maker's base had a detection ring (base DR in Figure 1) radius of roughly six grid units to use in monitoring the geographic space. The decision maker could detect the presence or absence of any track within this detection ring. Each base also had an identification ring (base IR in Figure 1) radius of roughly four grid units. A team member could discern whether a track was friendly or unfriendly once it was within this range. Any track outside the DR was invisible to the team member from the base. A team member who wanted to determine the nature of a track outside the identification ring had two options: ask teammates to share that information, or launch a vehicle and move it near the track. Since each vehicle had its own detection and identification rings and could be moved anywhere on the screen, all participants could detect and identify any track anywhere on the screen, but it took more effort to engage tracks outside of one's personal region.

Vehicles. Each team member had control of four vehicles that could be launched and moved to different areas of the screen. These vehicles could automatically perform certain functions (follow 
FIGURE 1

The DDD-MSU Grid

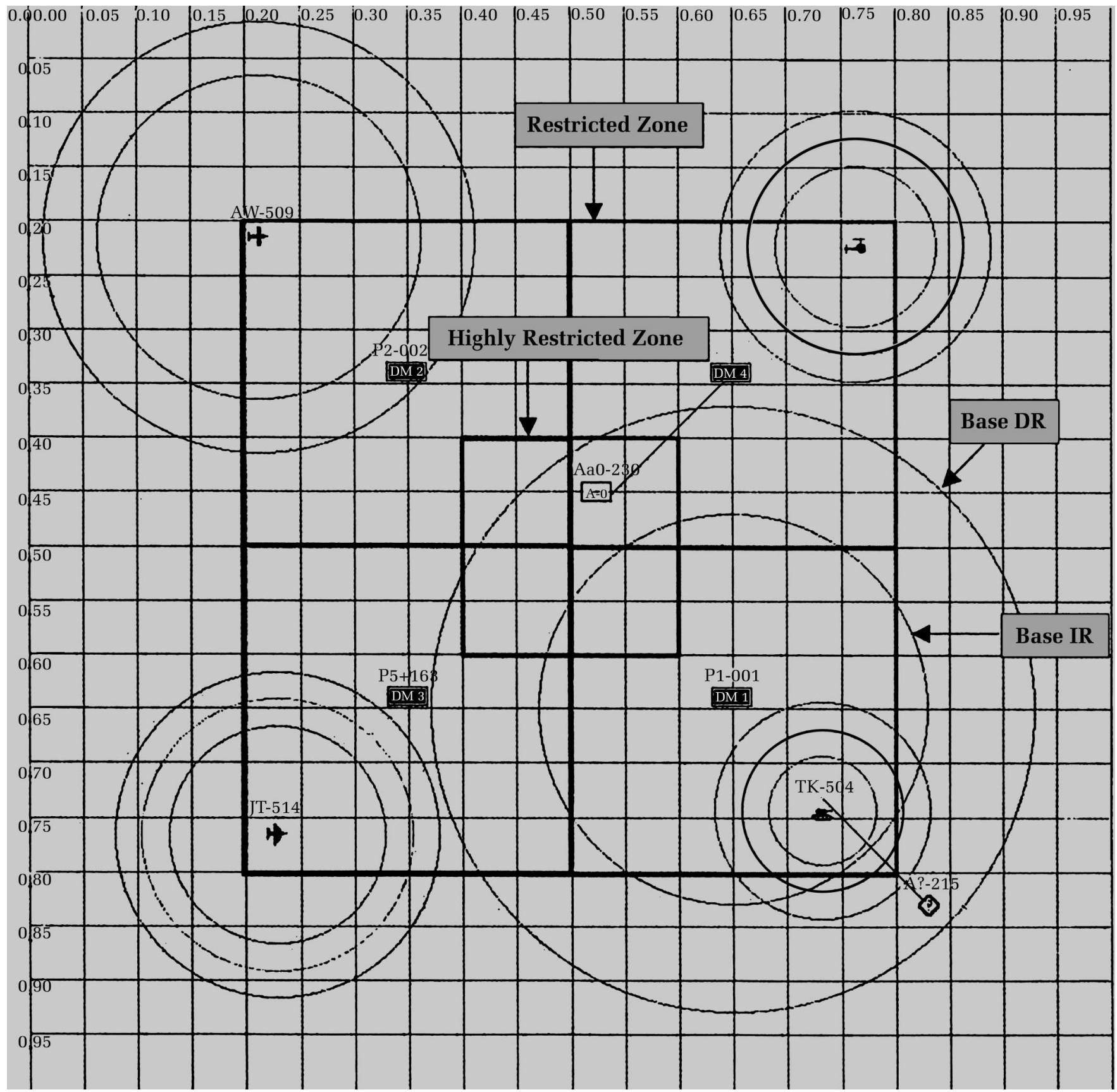

designated tracks, return to base to refuel, and so forth), and hence each team member was the manager of semi-intelligent agents. Each team member had one AWACS plane, one tank, one helicopter, and one jet. These vehicles varied in their capacities on four dimensions: range of vision, speed of movement, duration of operability, and weapons capacity.

An asset that was high on one dimension tended to be low on another, meaning each asset had its own unique advantages and disadvantages. For example, the tank had high weapons capacity but a short range of vision, whereas the AWACS had low weapons capacity but a wide range of vision. Thus, the various vehicles constituted a complex set of assets that ranged widely in their capacities. A symbol for each vehicle appears in Figure 1, along with the ranges of vision that characterized each vehicle (depicted by the largest ring surrounding each vehicle). A team member could operate any or 
all of the vehicles concurrently, but it took more effort to simultaneously operate multiple vehicles. For example, when a track appeared, a person could simply launch one vehicle and move it to engage the incoming track. Alternatively, the same person could work quickly to launch all four vehicles, move them to various areas of the geographic space, in anticipation of incoming tracks, and intercept them as soon as they crossed over into the restricted zone. Thus, the speed with which tracks were engaged was a function of how hard a person or team was working. Because of the variation in the four vehicles' capacities, it required a great deal of cognitive effort to effectively have all four vehicles out at once and then use them efficiently, but doing this did increase the speed with which tracks were engaged.

Identifying and engaging tracks. All tracks originated from the edge of the screen and proceeded inward. It was important for team members to identify tracks quickly and differentiate them along two dimensions: (1) friendly versus unfriendly and (2) standard versus novel. When a track was close enough to be detected but not close enough to be identified, it was represented by a question mark followed by a unique identification number set above a diamond (see the bottom right portion of Figure 1 for an example). Once the track came within the identification range of either the base or a vehicle, the team member could identify it. Once identified, the symbol representing the track changed from a diamond to a rectangle with a letter-number combination in it (see the middle of Figure 1). The letter indicated whether the track was in the air or on the ground. The number indicated whether the track was friendly or unfriendly, and if it was unfriendly, the amount of power needed to disable it. The team member who made the identification was the only one who could see this information, although he or she could share this information with other team members.

If a track within the restricted zones was identified as being unfriendly, team members needed to disable it. There were two requirements for successful disabling. First, the track had to be close enough, meaning that it had to be within the attack ring of the vehicle engaging it. Second, the vehicle needed to have as much power as the track (as indicated by the number in the rectangle), or more power. If a team member attempted to engage a track that was too far away or for which he or she had insufficient power, the track continued on unimpeded. If the track was successfully engaged, it disappeared from the screen. The attacking vehicle then had to return to base to reload and refuel.

There were eight types of "standard tracks" that were known a priori to have specific characteristics, and these were taught in the training session prior to the start of the task. There were also four types of "novel tracks" that were not encountered during training. Thus, team members did not know whether the novel tracks were air-based or groundbased, or friendly or unfriendly, or powerful or not powerful. Trial-and-error experience gained from the simulation was the only source of this knowledge. Thus, determining the nature of the novel tracks was a complex deductive exercise in which some behaviors were more diagnostic than others (better for supporting or refuting specific hypotheses about a track). This complexity created an opportunity for decision-making errors to occur, and thus the performance of teams could be evaluated not just in terms of their speed, but also in terms of their accuracy. Thus, a team's objective was to disable enemy tracks as fast as possible while not disabling any friendly tracks (that is, making friendly fire errors) or wasting resources by engaging enemy tracks with more power than was needed.

\section{Manipulations and Measures}

Reward structure. Teams were randomly assigned to either a cooperative or a competitive reward structure. Participants assigned to the cooperative condition were informed that each of the teams that had the best overall team performance would receive a reward of $\$ 40$, which would be split evenly among the team members, regardless of how well they performed as individuals. Participants under the competitive condition were informed that the top-performing individuals would each receive a reward of $\$ 10$, regardless of how well their teams performed as a whole.

Because the number of tracks was fixed at a relatively low number (19 tracks per quadrant), to obtain a score that was high enough to warrant winning the individual bonus in the competitive condition, an individual had to venture outside of his or her quadrant during the simulation. That is, he or she had to detect, identify, and attack tracks in the other team members' quadrants, thus limiting the potential score of the other team members. Thus, it was impossible for two members of one team to both qualify for the bonus in the competitive condition.

We used a four-item competitive orientation scale and a three-item cooperative orientation scale ( 1 = "disagree strongly" and $5=$ "agree strongly") to check the adequacy of the manipulation. A sample item used to measure competitive orientation was "While I was playing the DDD game, I was 
competing with the others on my team." A sample item used to measure cooperative orientation was "While I was playing the DDD game, it was important to achieve as many points as possible as a team." The four competitive items formed a reliable scale $(\alpha=.93)$, as did the three cooperative items $(\alpha=.93)$.

Extroversion and agreeableness. Extroversion and agreeableness were each measured with a 12item scale taken from the short form of the Revised NEO Personality Inventory (NEO-PI-R-short). This is the most widely used instrument for measuring the five-factor model, and Costa and McCrae (1992) have provided ample historical evidence on the reliability and construct validity of these measures. Coefficient alpha estimates of reliabilities for extroversion and agreeableness in this specific study were .79 and .76 , respectively.

If team members' extroversion and agreeableness are likely to be relevant for how teams react to reward structures, the question becomes how to aggregate these personality variables to the team level. In a recent review of empirical research on this issue, Moynihan and Peterson (2001) concluded that the best method of aggregating dispositional scores depends upon the nature of the task, particularly the degree of task interdependence. This conclusion is consistent with earlier arguments put forth by LePine, Hollenbeck, Ilgen, and Hedlund (1997), Barrick, Stewart, Neubert, and Mount (1998), and Neuman and Wright (1998), who all noted that when interdependence among task members is low, an additive model (using averages) is most appropriate, but when interdependence is high, a conjunctive model (using lowest scores) is most appropriate. According to Moynihan and Peterson, a conjunctive model better captures highly interdependent teams because it reflects the fact that one team member can have a disproportionate impact on a team as a whole. Because the level of interdependence among team members in MSU-DDD is clearly very high (see "Procedures"), we measured team composition using a conjunctive model.

Speed and accuracy. Speed was defined as the average amount of time it took to disable unfriendly tracks. Accuracy was success in avoiding two types of errors, both of which were automatically recorded by MSU-DDD. A friendly fire error occurred when a team member engaged a friendly track, and a rules of engagement error occurred when anyone engaged a track outside the restricted zone. The scores for each type of error were summed into an accuracy score.

Accuracy was then recoded so that a high value reflected high performance, and the values were then standardized to make the measures of the two task dimensions comparable. Speed and accuracy scores were assessed at both the team and individual level. Overall performance was measured as the sum of the standardized scores for speed and accuracy, also for both teams and individuals.

\section{Procedures}

We first administered the Revised NEO Personality Inventory to assess participants' extroversion and agreeableness. Then, each participant was randomly assigned to a four-person team, and then the teams were randomly assigned to reward structure conditions. The teams were trained together for approximately 90 minutes. Because rewards can only work if people have feedback and knowledge of results, we focused the team members on the relevant scores (individual scores in the competitive condition and team scores in the cooperative condition) throughout the training.

The first 30 minutes of training were devoted to familiarizing the participants with the object of the simulation, its scoring, and the capabilities and characteristics of the vehicles employed in the simulation. The next 30 minutes of training concentrated on how to manipulate the vehicles: launching them, moving them around the screen, identifying targets, and disabling targets. The final 30 minutes of training provided the participants with an opportunity to practice their new skills in an environment similar to the environment in which they would later perform. During this period, participants were allowed to ask their trainers questions as they practiced. In addition, the trainer could help those who seemed to be having difficulty with the task.

The teams then performed the task for the experimental session, during which each team, regardless of condition, experienced the same number, nature, timing, and sequence of tracks. Thus, the task was identical for all the teams. A total of 76 tracks appeared during the experimental session, and each participant experienced 19 tracks that originated in his or her quadrant. The tracks never stayed within the quadrant they originated in; instead, they crossed from one team member's area to another. It is also important to stress that the team members were not restricted to operating vehicles within their own quadrants, but instead could move their vehicles into other quadrants. Thus, even though a track may have originated in the SE quadrant, the team member from the NW quadrant could be the first person to engage it. Because both tracks and vehicles were free to roam quadrants, all teams in this simulation experienced a great deal of 
means interdependence. That is, what one person needed to do (or could do) was strongly influenced by what others were doing. For a team in the cooperative condition, if one team member was working slowly or ineffectively and failed to engage tracks that originated in his or her quadrant, all the other players had to "clean up after" this person. For a team in the competitive condition, if one person was aggressively "hogging all the tracks," the number of opportunities for other members of the team went down. Regardless of the team members' own perceptions of outcome interdependence, from a managerial perspective, the goal of each team was the same: defend the geographic space with as much speed and accuracy as possible.

\section{Data Analysis}

The research design employed in this study had both between-teams and within-teams elements. Reward structure was a between-teams measure, because each team obtained only one score ( 0 or 1$)$ for this variable. The nature of the task is a withinteams measure, since each team obtained two scores, one for speed and one for accuracy. Given this mixed-level design, we used repeatedmeasures regression analysis to analyze the data.

A full description of repeated-measures regression is beyond the scope of this article (see Cohen and Cohen [1983: 428-451] for an extensive treatment). In general, this type of analysis decomposes variance in the dependent variable (overall performance in this case) into two orthogonal sources; here, these are variability between teams (that is, some teams perform better than others regardless of the speed-accuracy distinction), and variability within teams (within a single team, there is variance in performance depending on whether speed or accuracy is the task dimension).

The criteria are then regressed on the predictors and matched to their levels. A between-teams manipulation like reward structure is used to try to explain variance between teams (some teams perform better than others regardless of the speedaccuracy distinction), whereas a within-teams measure like the nature of the task (the dummy-coded speed-accuracy distinction) is used to attempt to predict variance within teams (within any one team, performance varies depending upon whether one is looking at speed of task execution or accuracy of task execution). The technique also allows one to examine interactions among within- and between-teams measures and to directly test if the effect of a between-teams manipulation like reward structure has different influences on different task dimensions. Such a difference in effects is one of the core ideas underlying the contingency model we are testing.

The test of this within-between interaction is identical to what would be obtained if one were to treat speed and accuracy as separate dependent variables, separately regress reward structure on each, and then test for the differences in unstandardized regression coefficients. The repeatedmeasures approach, however, provides a direct measure of the statistical significance of the difference in unstandardized regression weights, as well as a direct measure of the effect size for the interaction (that is, the variance explained), the latter of which cannot be obtained when speed and accuracy are treated as separate dependent variables. Summarizing the above, we would note that if speed and accuracy were treated as separate dependent variables and analyzed in separate regression analyses, and if these analyses were followed up by a test for the differences in unstandardized regression coefficients, the conclusions drawn from our data would be identical to our present conclusions. However, we chose the repeated-measures regression approach because it was more parsimonious and direct (for more information on this topic, we refer the reader to Hollenbeck, Ilgen, and Sego [1994]).

\section{RESULTS}

\section{Descriptive Statistics and Manipulation Checks}

Table 1 presents the means, standard deviations, and correlations for the variables of interest. As might be expected, there was a slightly negative correlation between performance levels on the two dimensions of the task, speed and accuracy $(r=$ $-.22, p=.06$ ), suggesting some degree of trade-off. Also, as might be expected from the fact that extroversion and agreeableness are both parts of the interpersonal plane of the five-factor model circumplex, there was a slight positive correlation between these two variables $(r=.22, p=.06)$.

Analysis of variance (ANOVA) of cooperative versus competitive orientation showed that the manipulation of reward structure was successful. Teams working with the cooperative reward structure had a more cooperative orientation (mean = 4.26 , s.d. $=0.46$ ) than teams in the competitive reward structure $($ mean $=2.65$, s.d. $=0.53, F[1$, $73]=184.77, p<.01)$. Also, teams with the competitive reward structure had a more competitive orientation $($ mean $=3.51$, s.d. $=0.52)$ than teams in the cooperative reward structure (mean $=2.24$, s.d. $=0.53, F[1,73]=104.74, p<.01)$. 
TABLE 1

Descriptive Statistics for the Between-Team Variables ${ }^{a}$

\begin{tabular}{lcccccc}
\hline \multicolumn{1}{c}{ Variable } & Mean & s.d. & $\mathbf{1}$ & $\mathbf{2}$ & $\mathbf{3}$ & $\mathbf{5}$ \\
\hline 1. Reward structure & 0.40 & 0.49 & & & \\
2. Agreeableness & 3.10 & 0.47 & -.04 & .17 & & \\
3. Extroversion & 3.04 & 0.39 & $-.26^{*}$ & -.04 & -.03 & .22 \\
4. Speed & 0.00 & 1.00 & $.47^{*}$ & -.07 & -.22 \\
5. Accuracy & 0.00 & 1.00 & .16 & -.09 & .15 & $.65^{*}$ \\
6. Average performance & 0.00 & 0.63 & $.61^{*}$ \\
\hline
\end{tabular}

${ }^{\mathrm{a}} n=75$. Reward structure was dummy-coded; $0=$ competition and $1=$ cooperation. ${ }^{*} p<.05$

TABLE 2

Results of Repeated-Measures Regression Analysis for Performance on Task Dimension and Reward Structure ${ }^{\mathrm{a}}$

\begin{tabular}{|c|c|c|c|c|c|c|}
\hline Step & Independent Variable & $\boldsymbol{\beta}$ & Total $R^{2}$ & $\Delta R^{2}$ & $\begin{array}{c}\text { Incremental } \\
\text { Variance } \\
\text { within Teams }\end{array}$ & $\begin{array}{c}\text { Incremental } \\
\text { Variance } \\
\text { between teams }\end{array}$ \\
\hline 2 & Reward structure & -0.10 & .01 & .01 & & .03 \\
\hline 3 & $\begin{array}{l}\text { Task dimension } \times \text { reward } \\
\text { structure }\end{array}$ & $-1.29^{*}$ & $.14^{*}$ & $.13^{*}$ & $.21^{*}$ & \\
\hline
\end{tabular}

\footnotetext{
${ }^{\text {a }}$ For task dimension, speed $=0$ and accuracy $=1$. For reward structure, competition $=0$ and cooperation $=1$.

${ }^{\mathrm{b}} 61$ percent. $n=150$ (two observations per 75 teams; $d f=150-75-k-1$ ).

${ }^{\mathrm{c}} 39$ percent. $n=75$ (one observation per 75 teams; $d f=75-k-1$ ).

${ }^{*} p<.05$, one-tailed test
}

\section{Tests of Hypotheses}

Hypothesis 1. Table 2 shows the results of a repeated-measures regression analysis designed to test Hypothesis 1. This regression is based upon 150 observations: 75 teams were observed on two task aspects, speed and accuracy. As noted above, team-level variance in these 150 observations is either within-teams, based on the task dimension (speed versus accuracy) or between-teams, based on overall performance.

As shown in Table 2, 61 percent of the total variance in the 150 observations was attributable to within-teams variance, whereas the remaining 39 percent was attributable to between-teams variance. The first row of this table shows that there was no effect for speed versus accuracy (a natural result of standardizing the variables), and the second row shows that there was no "main effect" for reward structure (that is, no one structure was better irrespective of the speed-accuracy distinction). The third row of this table shows that a statistically significant interaction between the reward structure and the nature of the task explained 21 percent of the within-teams variance. This interaction is plotted in Figure 2. Consistent with Hypothesis 1, cooperative reward structures had a positive effect on accuracy, but a negative effect on speed, whereas competitive reward structures had a negative effect on accuracy, but a positive effect on speed. As noted above, if speed and accuracy were treated as separate dependent variables and analyzed in separate regression analyses, the conclusions drawn from our data would be identical to our present conclusions. Specifically, the coefficients associated with the separate regressions on speed and accuracy were -.26 and .47 , respectively ( $p=.03$ and .01 , respectively).

An additional analysis supported the idea that cooperative structures worked well because they promote diffusion of knowledge throughout a team. We analyzed accuracy scores separately for the standard and the novel tracks. (Recall that standard tracks were those covered in the training, and the novel tracks were not presented in training.) If the cooperative structure worked because it promoted diffusion of knowledge, then the effect this reward structure had on accuracy should be stronger for the novel tracks than for the standard tracks.

We tested this idea by creating a difference measure (novel-track errors minus standard-track errors) and comparing the scores between conditions. We found that the competitive teams made 3.58 
FIGURE 2

Interaction of Task Dimension and Reward Structure

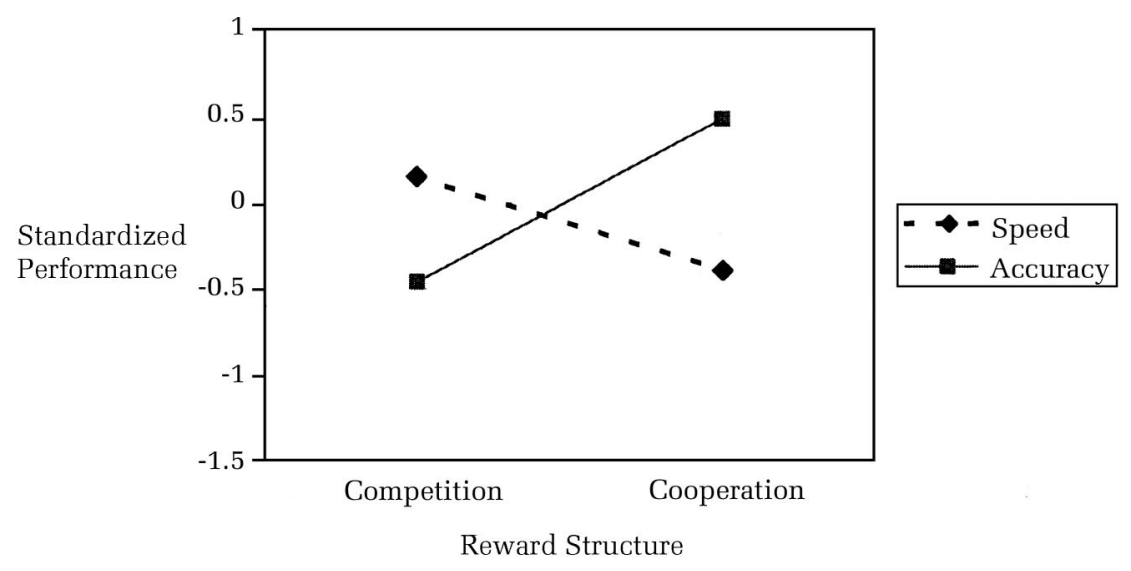

more novel-track errors than standard-track errors, but the cooperative teams only made 2.27 more novel-track errors than standard-track errors. This difference was significant $(t[73]=3.26, p<.01)$. Thus, our observations are consistent with goal interdependence theory: people working in cooperative reward structures seemed to do a better job of sharing information and diffusing knowledge about novel tracks that had to be gained on the job.

Hypotheses $2 a$ and $2 \boldsymbol{b}$. Table 3a shows the results of a regression equation designed to test Hypothesis 2a. The first row again shows that no single reward structure was best with respect to overall team performance aggregated across the two dimensions of the task. The second row of this table indicates that there was no main effect for extroversion. The third row of this table, however, indicates that the interaction between extroversion and the reward structure explained 5 percent of the variance. Figure 3, the plot of this interaction, shows that extroverts respond positively to cooperative reward structures, whereas introverts respond negatively to this type of reward structure. This pattern of findings supports Hypothesis 2a.

In Table $3 \mathrm{~b}$, results for the same equation computed for agreeableness mirror those in Table 3a in that there was no main effect for agreeableness, but

TABLE 3a

Results of Regression Analysis of Performance on Reward Structure and Extroversion ${ }^{a}$

\begin{tabular}{|c|c|c|c|c|}
\hline Step & Independent Variable & $\boldsymbol{\beta}$ & Total $R^{2}$ & $\Delta R^{2}$ \\
\hline 1 & Reward structure & 0.16 & .02 & .02 \\
\hline 2 & Extroversion & -0.12 & .04 & .02 \\
\hline 3 & Extroversion $\times$ reward structure & $1.75^{*}$ & $.09^{*}$ & $.05^{*}$ \\
\hline
\end{tabular}

a statistically significant interaction between agreeableness and reward structure. Figure 4 graphically shows that only agreeable individuals respond positively to cooperative structures. Thus, Hypothesis $2 \mathrm{~b}$ was supported.

The results documented in these tables are based upon a conjunctive measurement of team composition that was chosen because of the high level of means interdependence. Because the task could also be argued to have some additive elements, we repeated these regressions employing an additive model, and there were virtually no differences in the parameter estimates obtained from the alternative analysis.

In addition to evaluating results in terms of statistical significance, it is worthwhile to examine the sum of these effects in a practical sense, by looking at the raw number of decision-making errors made by different types of teams under different reward conditions. For the teams above the mean on extroversion and agreeableness and working under the cooperative reward structure, the average number of errors was 3.8. In contrast, under the competitive reward structure, teams above the mean on extroversion and agreeableness averaged 6.7 errors.

Hypothesis 3. Table 4 shows the results of two repeated-measures regression analyses designed to test Hypothesis 3. These regressions are replications of those presented in Table 2, with the exception that, rather than team-level performance, they examine the best and worst individual performance in each team. With respect to the poorest performer in each team, as shown in the top half of Table 4, 28 percent of the within-person variance in performance can be attributed to the interaction between reward structure and the task dimension. In contrast, as indicated in the bottom half of this table, 
FIGURE 3

Interaction of Extroversion and Reward Structure

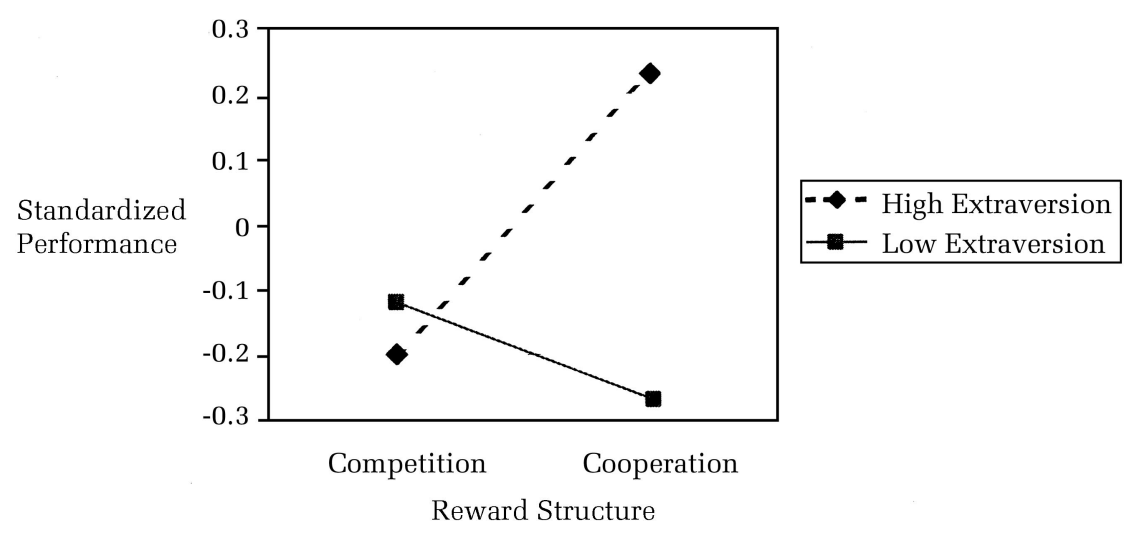

among the best performers this interaction explains only 9 percent of the variance. Figure 5 depicts these interactions; consistent with Hypothesis 3, although the interactions are similar for the best and the worst performers, they are clearly greater for the worst performers.

\section{DISCUSSION}

The current consensus regarding reward structures suggests that competitive structures should be used when people are working independently, whereas cooperative reward structures should be used when people are working interdependently (Deutsch, 1949; Miller \& Hamblin, 1963; Rosenbaum et al., 1980; Stanne et al., 1999; Wageman, 1995). Because all of the research participants in this study were working within means-interdependent teams, this study does not speak to how one should design reward structures when people are working alone. However, with respect to people who are working interdependently, the results from the current study both support and qualify the validity of the conventional recommendations.

\section{Reward Structures and Accuracy of Performance}

If a team is primarily concerned with the accuracy of performance, and if this team is composed

TABLE 3b

Results of Regression Analysis of Performance on Reward Structure and Agreeableness ${ }^{a}$

\begin{tabular}{|c|c|c|c|c|}
\hline Step & Independent Variable & $\boldsymbol{\beta}$ & Total $R^{2}$ & $\Delta R^{2}$ \\
\hline 1 & Reward structure & 0.16 & .02 & .02 \\
\hline 2 & Agreeableness & 0.08 & .03 & .01 \\
\hline 3 & Agreeableness $\times$ reward structure & $1.63^{*}$ & $.08^{*}$ & $.05^{*}$ \\
\hline
\end{tabular}

of extroverted and agreeable members, our results validate the conventional recommendations. That is, when it came to decision-making errors, a team with the appropriate interpersonal orientation just described made almost twice as many errors when placed in a competitive reward structure than the same type of team placed in a cooperative reward structure.

\section{Reward Structures and Speed of Performance}

Few teams have an unlimited amount of time in which to complete their work and, in line with past research (Elliott et al., 2001; Woodworth, 1899), this study showed that speed and accuracy are separable aspects of a task $(r=-.22)$. Moreover, when it came to decomposing variance in team performance, most of the variance was attributable to within-team differences on the two different aspects of the task (61\%), not to between-team differences in overall performance $(39 \%)$. If one recognizes this distinction, the standard recommendation regarding the use of cooperative rewards in interdependent teams needs some qualification.

Cooperative reward structures had a negative effect on the teams' speed. The measurable impact of this interaction far exceeded what one would expect just given the moderate negative relationship between the two performance dimensions. That is, although accuracy alone accounted for only 5 percent of the variance in speed, the interaction of reward structure and task dimension accounted for 21 percent of the variance. This seems to suggest that there was something slowing teams down in the cooperative structure other than simply the greater attention they were giving to accuracy.

The interaction plotted in Figure 5 suggests that this finding may be attributable to social loafing on the part of the worst performer. As is apparent in this figure, the reward structure had virtually no 
FIGURE 4

Interaction of Agreeableness and Reward Structure

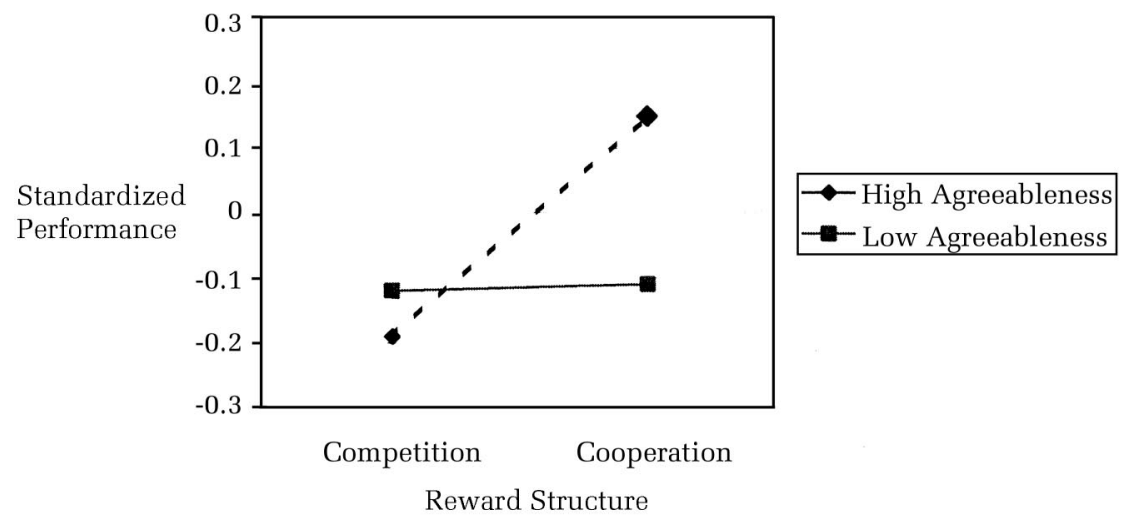

effect on the speed of the best performer, but a discernable effect on the speed of the slowest team member. If the effect of cooperative reward structures on speed simply reflected more time spent on discussion, information sharing, and a concern for accuracy, this timing effect should have been in evidence for both the best and worst performers. After all, according to goal interdependence theory, these two people should both be talking-in fact, they should be talking to each other. Instead, only the worst performer seemed to slow down when a free-riding opportunity was created by the cooperative reward structure. As the social loafing research would suggest, switching from a cooperative to a competitive reward structure largely solved the speed problem in these teams.

Although few organizations would be willing to forego all quality considerations in a full-blown effort to speed workers up, it is nevertheless true that many organizations compete on speed. In fact, in many negotiations, delivery time is a deciding factor in successfully winning contracts (road construction teams are an example). In addition, in certain contexts (such as seasonal promotions in a retail unit), unless the product can be delivered on time, its quality will not matter. Speed of operations is also a well-known force multiplier: if the production of given number of workers in a manu-

TABLE 4

Results of Repeated-Measures Regression Analysis of Performance on Task Dimension and Reward Structure for the Worst and Best Performers

\begin{tabular}{|c|c|c|c|c|c|c|}
\hline Step & $\begin{array}{c}\text { Independent } \\
\text { Variable }\end{array}$ & $\boldsymbol{\beta}$ & $\begin{array}{c}\text { Total } \\
R^{2}\end{array}$ & $\Delta R^{2}$ & $\begin{array}{c}\text { Incremental } \\
\text { Variance within } \\
\text { Teams }^{\mathbf{a}}\end{array}$ & $\begin{array}{c}\text { Incremental } \\
\text { Variance } \\
\text { between Teams }\end{array}$ \\
\hline 1 & Task dimension & 0.04 & .00 & .00 & .00 & \\
\hline 2 & $\begin{array}{l}\text { Reward } \\
\text { structure }\end{array}$ & $-0.20^{*}$ & .04 & .04 & & $.09^{*}$ \\
\hline 3 & performers & & & & & \\
\hline 1 & Task dimension & -0.09 & .01 & .01 & .02 & \\
\hline 2 & $\begin{array}{l}\text { Reward } \\
\text { structure }\end{array}$ & -0.14 & .03 & .02 & & .04 \\
\hline
\end{tabular}

\footnotetext{
${ }^{\text {a }} n=150$ (two observations per 75 teams; $d f=150-75-k-1$ ).

${ }^{\mathrm{b}} n=75$ (one observation per 75 teams; $d f=75-k-1$ ). Between-teams variance is 46 percent for the worst performers and 45 percent for the best performers.

${ }^{*} p<.05$, one-tailed test
} 
FIGURE 5

Interaction of Task Dimension, Reward Structure, and Level of Performance

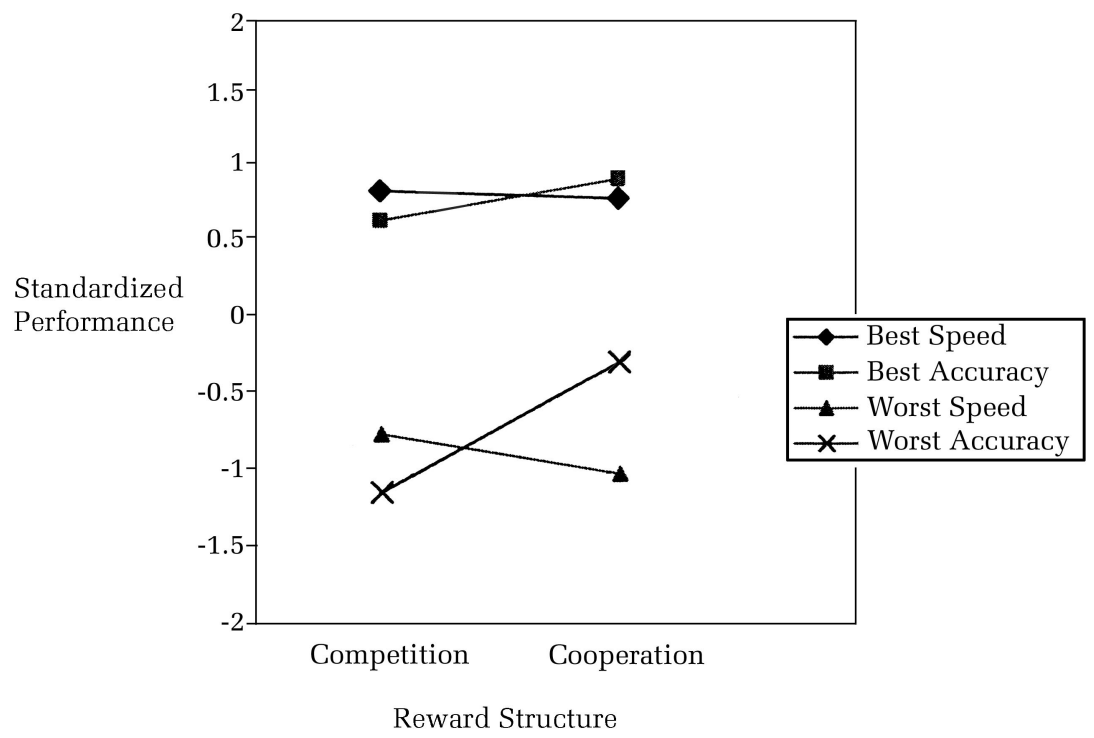

facturing team can be doubled, the size of the team can be cut in half. Thus, speed of operations is critical to organizations that are competing on the basis of cost rather than differentiation.

Finally, in product development teams, a typical product development cycle often requires a focus on quality rather than speed in the beginning. In the initial stages of product development, quality may be critical to creating a small niche market for a new product, and the lack of rival products provides the development team the luxury of not having to worry about speed or cost. However, as the product matures and becomes standardized, more competitors may enter the market, and the speed of production becomes more critical. Thus, the changes that take place over product life cycles may require an evolution from collaborative reward structures that place little premium on speed in the beginning to more competitive schemes, in which speed and cost are the central driving factors, at the end of the cycle.

In addition to these types of external considerations, the need to focus on speed is also critical for internal team dynamics. If some workers are working feverishly toward accomplishing a team's mission while others are taking it easy, it is only a matter of time before perceptions of inequity create negative interaction patterns that could threaten the team's viability (Ezzamel \& Willmot, 1998). For all these reasons, while recognizing the virtues of cooperative structures with respect to promoting quality, we believe that recognizing the liabilities of these structures when it comes to speed is also essential.

\section{Reward Structures and Interpersonal Orientation}

Just as no one reward structure is best for both dimensions of team tasks, there is no one best way to design reward structures irrespective of team composition. Past studies that have searched for interactions between individual differences and reward structures have met with little success (Wageman, 1995), but their lack of success may be attributable to the use of locally developed individual difference measures of unknown validity. In this study, we used the well-accepted five-factor model framework and the extensively documented NEO-PI instrument (Costa \& McCrae, 1992) to characterize team composition and found significant interaction effects between reward structure and personality variables.

Specifically, we found that both aspects of the interpersonal orientation domain of the five-factor model circumplex were relevant for predicting how teams composed of different types of people reacted to various reward structures. Goal interdependence theory is based upon the presumption that cooperative reward structures promote collaboration and trust, but the five-factor model clearly denotes that there are stable individual differences in the degree to which people are naturally collaborative (that is, extroverted) and trusting (that is, agreeable). Our study is the first research attempt to see how people react when placed in a reward structure that either reinforced or contradicted their natural proclivities.

The results indicated that the conventional recommendation derived from goal interdependence 
theory regarding the use of cooperative reward structures in interdependent teams was validated in extroverted and agreeable teams. However, this same reward structure did not promote performance when it contradicted the natural tendencies of teams (that is, when teams were low on agreeableness and extroversion). A close examination of Figures 3 and 4, however, indicates that although teams comprised of introverted and disagreeable members clearly did not respond positively to cooperative reward structures, they did not in fact respond all that well to competitive structures either. Apparently, the value in creating a good fit between the people and the reward structure was much greater for agreeable extroverts than it was for disagreeable introverts.

The disagreeable introverts in this study clearly took exception to the cooperative reward structure that yoked their outcomes to those of others. However, they may have also found both the high level of means interdependence inherent in this task and the negative outcome relationship created by the competitive reward structure somewhat objectionable. In other words, disagreeable introverts may not want to work in teams at all, and rather than being put in competition with others, would rather just be left alone.

One might be tempted to design work for these types of people as independent jobs. However, setting tasks up as series of noninteracting individual jobs is also problematic. As is the case with many complex team tasks, uncertainty was inherent in the workload distribution in the simulation used in this study. At any one time, a certain team member might be flooded with tracks, but at other times, there might be very little activity in this person's section. The reason many organizations use teams in the first place is that team-based structures enable dynamic workload adjustment. Team members who have few tasks on their hands can go and help others who are busy. If one were to "deteam" the task used in the current study by, for example, saying that no team member could leave his or her region, overall performance would be harmed because dynamic workload adjustment would be prevented. Therefore, future research needs to explore how to best structure work and rewards for people who are low in agreeableness and extroversion.

Another interesting direction for future research would be to investigate the impact of structures in which multiple rewards are used, some of which are allocated cooperatively whereas others are allocated competitively. When there is only one single reward, cooperative and competitive structures are mutually exclusive ends of a continuum, but when there are multiple rewards, one can take a cooper- ative approach with one type of reward (such as an end-of-the-year team bonus), but a competitive approach to another type of reward (such as an endof-the-year merit pay raise). Although questions like these are beyond the scope of the current study, it would be interesting to see how the combination of the two different reward systems would play out (for instance, does one trump the other, do they each neutralize each other, or do individual differences take over?). Future experiments comparing cooperative and competitive reward structures could be efforts to answer these questions by adding a condition in which the two reward structures are combined (by rewarding participants with both an individual base pay and a collaborative team-based bonus). It might also be interesting to study reward structures that change from one type to the other, to see how the dynamics of changing reward structures evolve over time.

\section{Practical Implications}

Many complex tasks embody a speed-accuracy trade-off; thus, these findings, which suggest that cooperative rewards promote accuracy, whereas competitive rewards promote speed, have important implications for practice. Managers may not be able to jointly maximize both aspects of task performance via a single reward structure, and thus they should consider which aspect of a task they want to prioritize before designing the reward structure.

Second, our findings have important practical implications for two types of questions regarding team composition. Organizations may pose the question of which people should be selected to compose a team. Our findings show that if cooperative reward structures are in place, teams perform much better when composed of people who are high on extroversion and agreeableness. Organizations also may pose the question of which reward structure works best with a given team. That is, if a team's members have already been selected and are in place, what reward structure should be applied? Our findings show that when teams are composed of extroverted and agreeable members, a cooperative reward structure is a very effective choice.

\section{Limitations}

The fact that this study was conducted in a laboratory context may evoke the usual questions regarding the external validity of the findings. Participants in this study were not randomly selected from any definable population, but rather were college students. One disadvantage that this procedure entails is that the sample of subjects that partici- 
pated in our study was culturally quite homogeneous and, therefore, we cannot be certain that our findings generalize to different populations. Examining the impact of cultural factors on how reward structures influence team performance would be an interesting question for future research, because the possibility exists that reward structures interact with cultural factors.

A second limitation that also concerns the external validity of our findings relates to the task used in the current study. Although we believe that this task is representative of many kinds of meansinterdependent team tasks that have a speed-accuracy trade-off (including the tasks that manufacturing teams, emergency medical teams, pit crews, air traffic controllers, and weapons directors need to perform), we technically cannot generalize the parameter estimates found in this study to all other tasks, because we did not randomly select the task from the entire population of team tasks. However, prior research has shown that participants who engage in the task we used in the current study do find it psychologically engaging. Moreover, they were aware of the financial bonuses that could be achieved by performing well on the task and were genuinely interested in winning the bonus money. Indeed, if anything, the meta-analytic evidence regarding incentives suggests that in almost all cases, the findings from laboratory contexts provide conservative estimates of what is found in field settings using the analogous interventions (Jenkins et al., 1998).

Moreover, the primary purpose of this study was to test the boundary conditions of goal interdependence theory and, for the most part, research on this theory is based upon similarly structured studies. Nothing inherent in the theory implies that it would not work in the current context, suggesting that this context is a viable one in which to test this theory. Indeed, it would be very difficult to rigorously test many of the ideas tested here in a field setting. Given what is known about the attractionselection-retention cycle, a field study that allowed natural gravitation of people to teams, reward structures, and tasks would be highly confounded. Moreover, subjective supervisory judgments regarding speed and accuracy of performance are notoriously unreliable and low in discriminant validity. Thus, in order to draw rigorous causal inferences in this context, it is crucial to (1) randomly assign people to teams, (2) randomly assign teams to conditions, (3) create objectively identical task demands, and (4) obtain objective measures of accuracy and speed. Nevertheless, a field study that could overcome these traditional difficulties could shed some light on the nuances that make the ap- plication of either of these two types of reward structures more difficult in practice than it might seem in theory it would be.

A third limitation here is that the experimenters conveyed the manipulations and thus were not blind to experimental conditions. Although we took extreme care to write protocols that stated the exact words an experimenter had to say to participants at various points during the experiment, we cannot exclude the possibility that the experimenters influenced the data in some subtle way, through their nonverbal behavior, for example. Future research could address this limitation of the current study by employing alternative means of providing the information regarding reward structures.

A final aspect of the current study that may be viewed as a limitation is that the manipulation of reward structure was reinforced; team members in the competitive reward structure condition were told that during the task, that they should pay attention to their own individual scores, whereas team members in the cooperative reward structure condition were told that they should pay attention to their team's score. We focused the team members on the relevant scores throughout the training to make sure they understood the reward structure under which they worked, because rewards can only work if people have feedback on and knowledge of results. In most real-life situations, reward structures co-occur with a performance monitoring system that matches the reward structure (for example, it would not make sense to tell a team's members they will be rewarded for their individual performance and then measure the performance of the team as a whole). The same was true for our study. Therefore, focusing the subjects on the "right" scores was an important part of our manipulation. However, critical readers may ask whether it was our manipulation of reward structure per se, or the participants' focus on the relevant scores, or the combination of both that caused the effects reported here. Future research should address this issue by separately manipulating reward structure and the scores participants focus on during an experiment.

\section{Directions for Future Research}

The current study focused on moderators of the effects of reward structures, not mediators of the effects of reward structures. We focused first on these moderating variables because, from an applied perspective, establishing the factors that influence the relationship between reward structure and performance is key in answering questions about which structure to use in which contexts 
with which people. However, this focus on moderation limits our ability to answer the mediation question that might be of interest from an academic perspective. Teams working under a cooperative reward structure may have outperformed competitive teams on accuracy for a number of reasons, and the same can be said for the effects of competitive structure on speed. This study cannot pin down the precise mechanisms underlying all of the effects detected here. While admitting this, we note that we hope that researchers, armed with the knowledge provided by this study (for instance, that different reward structures work best under different conditions), may be able to extend this work by isolating the precise reason for these effects in a more direct way. This latter type of effort will only be forthcoming, however, if future researchers realize that there are interesting interactions between reward structures and conditions regarding the dimensions of a task, the personality composition of teams, and individual performance levels within teams, that need to be explained.

\section{REFERENCES}

Allred, B. B., Snow, C. C., \& Miles, R. E. 1996. Characteristics of managerial careers in the 21st century. Academy of Management Executive, 10(4): 17-27.

Barrick, M. R., Stewart, G. L., Neubert, M. J., \& Mount, M. K. 1998. Relating member ability and personality to work-team processes and team effectiveness. Journal of Applied Psychology, 83: 377-391.

Beersma, B., \& De Dreu, C. K. W. 2003. The aftermath of group negotiation: How social motives affect distal group functioning and performance. Working paper, University of Amsterdam.

Cohen, J., \& Cohen, P. 1983. Applied multiple regression/correlation analysis for the behavioral sciences. Mahwah, NJ: Erlbaum.

Costa, P. T., \& McCrae, R. R. 1992. Revised NEO personality inventory. Odessa, FL: Psychological Assessment Resources.

Deutsch, M. 1949. A theory of cooperation and competition. Human Relations, 2: 129-152.

Elliott, D., Helsen, W. F., \& Chua, R. 2001. A century later: Woodworth's (1899) two-component model of goal directed aiming. Psychological Bulletin, 127: 342-357.

Ezzamel, M., \& Willmot, H. 1998. Accounting for teamwork: A critical study of group-based systems of organizational control. Administrative Science Quarterly, 43: 358-395.

Hackman, J. R. 1998. Why teams don't work. In S. R. Tindale \& L. Heath (Eds.), Theory and research on small groups: 245-265. New York: Plenum.
Hollenbeck, J. R., Ilgen, D. R., Moon, H., Sheppard, L., Ellis, A., West, B., \& Porter, C. O. L. H. 2002. Structural contingency theory and individual differences: Examination of external and internal person-team fit. Journal of Applied Psychology, 87: 599-606.

Hollenbeck, J. R., Ilgen, D. R., \& Sego, D. J. 1994. Repeated measures regression and mediational tests: Enhancing the power of leadership research. Leadership Quarterly, 5: 3-23.

Ivancevich, J. M., \& Matteson, M. T. 1999. Organizational behavior and management (5th ed.). Boston: McGraw-Hill.

Jenkins, G. D., Mitra, A., Gupta, N., \& Shaw, J. D. 1998. Are financial incentives related to performance? A meta-analytic review of empirical research. Journal of Applied Psychology, 83: 777-787.

Kristof, A. 1996. Person-organization fit: An integrative review of its conceptualization, measurement and implications. Personnel Psychology, 49: 1-49.

Latane, B., Williams, K., \& Harkins, S. G. 1979. Many hands make light the work: The causes and consequences of social loafing. Journal of Personality and Social Psychology, 37: 822-832.

LePine, J. A., Hollenbeck, J. R., Ilgen, D. R., \& Hedlund, J. 1997. Effects of individual differences on the performance of hierarchical decision-making teams: Much more than g. Journal of Applied Psychology, 5: 803811.

LePine, J. A., \& Van Dyne, L. 2001. Peer responses to low performers: An attributional model of helping in the context of groups. Academy of Management Review, 26: 67-84.

Levine, J. M., \& Moreland, R. L. 1998. Small groups. In D. Gilbert, S. Fiske, \& G. Lindzey (Eds.), Handbook of social psychology, (4th ed.), vol. 2: 415-469. Boston: McGraw-Hill.

McCrae, R. R., \& Costa, P. T. 1997. Personality trait structure as a human universal. American Psychologist, 52: 509-516.

McDougall, W. 1932. Of the words character and personality. Character and Personality, 1: 3-16.

Miles, J. A., \& Greenberg, J. 1993. Using punishment threats to attenuate social loafing effects among swimmers. Organizational Behavior and Human Decision Processes, 56: 246-265.

Miller, L. K., \& Hamblin, R. L. 1963. Interdependence, differential rewarding, and productivity. American Sociological Review, 28: 768-778.

Moynihan, L. M., \& Peterson, R. S. 2001. A contingent configuration approach to understanding the role of personality in organizational groups. In R. I. Sutton \& B. M. Staw (Eds.), Research in organizational behavior, vol. 23: 327-378. Greenwich, CT: JAI Press.

Neuman, G. A., \& Wright, J. 1999. Team effectiveness: 
Beyond skills and cognitive ability. Journal of Applied Psychology, 84: 376-389.

Rosenbaum, M., Moore, D., Cotton, J., Cook, M., Heiser, R., Shovar, N., \& Gray, M. 1980. Group productivity and process: Pure and mixed reward structures and task interdependence. Journal of Personality and Social Psychology, 39: 626-642.

Stanne, M. B., Johnson, D. W., \& Johnson, R. T. 1999. Does competition enhance or inhibit motor performance: A meta-analysis. Psychological Bulletin, 125: 133-154.

Wageman, R. 1995. Interdependence and group effectiveness. Administrative Science Quarterly, 40: 145180.

Woodworth, R. S. 1899. The accuracy of voluntary movement. Psychological Review, 3: 1-119.

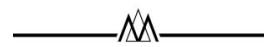

Bianca Beersma (b.beersma@uva.nl) is a postdoctoral fellow in organizational psychology at the University of Amsterdam. She received her doctorate from the University of Amsterdam. Her research interests include team performance, social motives in teams, group negotiations, and conflict management.

John R. Hollenbeck received his Ph.D. in management from New York University in 1984, and he is currently the Eli Broad Professor of Management at the Eli Broad Graduate School of Business Administration at Michigan
State University. He cofounded the Michigan State University Team Effectiveness Research Laboratory; the multilevel theory of team decision making grew out of the program of research there. Professor Hollenbeck is also the codeveloper of the most highly cited model and measure of goal commitment, one of the central features of all self-regulation theories of motivation.

Stephen E. Humphrey is a doctoral candidate in organizational behavior at the Eli Broad Graduate School of Management at Michigan State University. His research interests include teams and the group context, decision making, and dispositions.

Henry Moon is an assistant professor at Emory University. He received his Ph.D. at Michigan State University. He studies decision making, teams, and personality.

Donald E. Conlon is a professor of management at the Eli Broad Graduate School of Management, Michigan State University. He received his Ph.D. in business administration from the organizational behavior group at the University of Illinois. His current research interests include organizational justice theory, negotiation and conflict management, and managerial decision making.

Daniel R. Ilgen is the John A. Hannah Distinguished Professor of Psychology and Management at Michigan State University. He received his Ph.D. from the University of Illinois. The cofounder of the Michigan State University Team Effectiveness Research Laboratory, he has written extensively on work motivation and team decision making. 
Copyright of Academy of Management Journal is the property of Academy of Management and its content may not be copied or emailed to multiple sites or posted to a listserv without the copyright holder's express written permission. However, users may print, download, or email articles for individual use. 